\title{
Intralymphatic immunotherapy: a 3-year randomized, double-blind study in 72 patients with allergic rhinitis due to birch and grass.
}

\author{
Lars Ahlbeck ${ }^{1}$, Emelie Ahlberg ${ }^{2}$, Janne Björkander ${ }^{3}$, Caroline Aldén², Georgia \\ Papapavlou ${ }^{2}$, Laura Palmberg ${ }^{2}$, Ulla Nyström ${ }^{1}$, Pavlos Retsas ${ }^{1}$, Patrik Nordenfelt ${ }^{3}$, Totte \\ Togö ${ }^{1}$, Pål Johansen ${ }^{4}$, Bo Rolander ${ }^{5}$, Maria Jenmalm² ${ }^{2}$, and Lennart Nilsson ${ }^{1}$ \\ ${ }^{1}$ Linköping University Hospital \\ ${ }^{2}$ Linköping University \\ ${ }^{3}$ Ryhov County Hospital \\ ${ }^{4}$ University Hospital Zurich \\ ${ }^{5}$ Futurum Academy of Health and Care
}

August 6, 2020

\begin{abstract}
Background: There is need for a fast, efficient, and safe way to induce tolerance in patients with allergic rhinitis. Methods: Patients with birch and timothy allergy were randomized and received three doses of $0.1 \mathrm{ml}$ of birch and 5 -grass allergen extracts (10,000 SQ units/ml, ALK-Abelló), or birch and placebo or 5-grass and placebo by ultrasound-guided injections into inguinal lymph nodes at monthly intervals. Rhinoconjunctivitis Total Symptom Score, Medication Score and Rhinoconjunctivitis Quality of Life Questionnaire were evaluated before treatment and after each birch and grass pollen season during three subsequent years. Circulating proportions of $\mathrm{T}$ helper subsets and allergen-induced cytokine and chemokine production were analyzed by flow cytometry and Luminex. Results: The three groups reported fewer symptoms, lower use of medication and improved quality of life during the birch and grass pollen seasons each year after treatment at an almost similar rate independently of treatment. Nine patients had severe adverse events which were judged to be unrelated to the therapy. Mild local pain was the most common adverse event. IgE levels to birch decreased, whereas birch-induced IL-10 secretion increased independently of treatment. IgG4 levels to birch and timothy and skin prick test reactivity remained mainly unchanged. Conjunctival challenge tests with timothy extract showed a higher threshold for allergen. In all three groups, regulatory $\mathrm{T}$ cell frequencies were increased three years after treatment. Conclusion: Intralymphatic immunotherapy against grass and birch pollen allergy was effective, safe and associated with bystander immune modulatory responses.
\end{abstract}

Manuscript without references, no of words: 3469

\section{Abstract}

Background: There is need for a fast, efficient, and safe way to induce tolerance in patients with allergic rhinitis.

Methods: Patients with birch and timothy allergy were randomized and received three doses of $0.1 \mathrm{ml}$ of birch and 5-grass allergen extracts (10,000 SQ units/ml, ALK-Abelló), or birch and placebo or 5-grass and placebo by ultrasound-guided injections into inguinal lymph nodes at monthly intervals. Rhinoconjunctivitis Total Symptom Score, Medication Score and Rhinoconjunctivitis Quality of Life Questionnaire were evaluated before treatment and after each birch and grass pollen season during three subsequent years. Circulating 
proportions of $\mathrm{T}$ helper subsets and allergen-induced cytokine and chemokine production were analyzed by flow cytometry and Luminex.

Results: The three groups reported fewer symptoms, lower use of medication and improved quality of life during the birch and grass pollen seasons each year after treatment at an almost similar rate independently of treatment. Nine patients had severe adverse events which were judged to be unrelated to the therapy. Mild local pain was the most common adverse event. IgE levels to birch decreased, whereas birch-induced IL-10 secretion increased independently of treatment. IgG4 levels to birch and timothy and skin prick test reactivity remained mainly unchanged. Conjunctival challenge tests with timothy extract showed a higher threshold for allergen. In all three groups, regulatory $\mathrm{T}$ cell frequencies were increased three years after treatment.

Conclusion: Intralymphatic immunotherapy against grass and birch pollen allergy was effective, safe and associated with bystander immune modulatory responses.

Trial registration: EudraCT (2013-004726-28).

Keywords: allergy treatment, immunotherapy clinical, T-cells, quality-of-life

\section{Abbreviations:}

AE Adverse event

AIT Allergen immunotherapy

aTreg Activated T regulatory cell

CAPT Conjunctival allergen provocation test

CCL17 CC motif chemokine ligand 17

CXCL10 CXC motif chemokine ligand 10

FENO Fraction of exhaled nitric oxide

FEV1\% Forced expiratory volume at the end of the first second, percent of predicted value

FoxP3 Forkhead box P3

GATA3 GATA binding protein 3

ILIT Intralymphatic immunotherapy

IL-4 Interleukin-4

MS Medication score

PEF Peak expiratory flow

RORC Retinoic-acid related orphan receptor C

RQLQ Rhinoconjunctivitis quality of life questionnaire

RTSS Rhinoconjunctivitis total symptom score

rTreg Resting $\mathrm{T}$ regulatory cell

SPT Skin prick test

SCIT Subcutaneous immunotherapy

SLIT Sublingual immunotherapy

SQ-U Standardized quantified units 
Tbet T-box expressed in $\mathrm{T}$ cell

Th T helper

Treg T regulatory cell

U-HCG Urine human chorionic gonadotropin

\section{Introduction}

Nearly $30 \%$ of the adult population of Sweden report allergic rhinitis ${ }^{1}$. In addition, the prevalence of allergic sensitization is up to $50 \%$ in Sweden and in many other European countries ${ }^{2,3}$. The total cost of allergic rhinitis in Sweden, with a population of 9.5 million (in 2014), has been estimated at \euro1.3 billion annually ${ }^{4}$. Treating these patients with allergen immunotherapy (AIT) is cost-effective ${ }^{5}$. To date, AIT is the only treatment that affects the long-term development of allergic rhinoconjunctivitis. It induces clinical tolerance primarily by stimulating regulatory $\mathrm{T}$ (Treg) cells, attenuating $\mathrm{T}$ helper 2 (Th2) responses and inducing blocking antibodies ${ }^{6}$. Conventional AIT with subcutaneous injections is effective, but consumes time and resources ${ }^{7,8}$. Therefore, despite its advantages in allergic rhinitis, AIT has not achieved its full potential. In Sweden, only 15/10,000 receive this treatment, and figures vary worldwide ${ }^{9}$.

AIT improves symptom, medication and combined symptom and medication scores (MS) in patients with allergic rhinoconjunctivitis ${ }^{10}$. A randomized placebo-controlled trial with subcutaneous immunotherapy (SCIT) with birch pollen allergen reduced the symptom score by $40 \%$ over placebo ${ }^{11}$. In a review article of SCIT and sublingual immunotherapy (SLIT) trials, SCIT was reported to reduce nasal and ocular symptoms by $32-36 \%$ compared with placebo, whereas SLIT produced a reduction of $26-36 \%$ compared with placebo ${ }^{12}$. In an open intralymphatic immunotherapy (ILIT) study, hay-fever patients received only three monthly allergen injections directly into the inguinal lymph nodes and an accumulated dose of only 3,000 standardized quantified units (SQ-U) in contrast to approximately 3,000,000 SQ-U with SCIT. Greater efficacy and safety, and faster relief of symptoms were observed after ILIT than after SCIT ${ }^{13}$. The study was followed by a few smaller studies of which five ${ }^{14-18}$ confirmed the conclusion from the original trial, whereas one showed no benefit of ILIT ${ }^{19}$. Recently we performed a small open pilot study of 10 patients treated with ILIT for birch or grass pollen allergy. We concluded that ILIT was associated with improved quality of life, reduced symptoms, and beneficial immunological changes ${ }^{18}$. However, further studies are required to determine whether ILIT can induce clinical effects similar to those of SCIT concerning rhinitis symptoms and how ILIT affects immune responses ${ }^{20}$.

The objective was to evaluate whether ILIT can be an alternative to SCIT and SLIT. We also aimed to determine its safety, efficacy and immune modulatory effects, including changes in spontaneous and allergeninduced cytokine and chemokine production, and proportions of circulating $\mathrm{T}$ helper cell subsets.

\section{Methods}

\section{Study design}

A 3-year double-blind randomized clinical trial in 72 patients with rhinoconjunctivitis due to sensitization with birch and grass pollen allergens.

\section{Study population eligibility criteria}

In all, 126 patients were assessed for eligibility. Forty-four did not meet the inclusion criteria, 7 withdrew consent before treatment and 1 was excluded for unknown reasons (Fig. 1). Fifty-seven patients with allergic rhinoconjunctivitis due to birch and timothy pollen allergens were randomized in 2014 and 17 patients 2015. The 74 patients, including 35 females, were 19-53 years old and had seasonal allergic symptoms to birch and grass (Table 1). Their skin prick test was $>3 \mathrm{~mm}$ and displayed IgE to birch and timothy $>0.35 \mathrm{kU} / \mathrm{L}$. Exclusion criteria were pulmonary disease, $<80 \%$ of predicted forced expiratory volume at the end of the first second (FEV1), use of more than $800 \mu \mathrm{g}$ inhaled budesonide (or equivalent) per day, pregnancy, severe arterial hypertension, autoimmunity, cardiovascular, hepatic, renal, upper airway or metabolic disease, 
mental incapacity, alcohol abuse, medication interfering with immune response or beta-blockers. From earlier studies we expected 8 out of 9 patients would improve at least $40 \%$. With 40 active treated and 20 in the placebo group, with an alpha of 0.05 the power was calculated to $92 \%$.

\section{Intralymphatic immunotherapy}

The patients were randomized into three groups receiving three doses at four-week intervals of $0.1 \mathrm{ml}$ of birch pollen allergen on aluminum hydroxide (10,000 SQ-U/ml; ALK-Abelló, Hørsholm, Denmark) and/or $0.1 \mathrm{ml}$ of 5-grass pollen allergen on aluminum hydroxide (10,000 SQ-U/ml; ALK-Abelló, Hørsholm, Denmark. 5-grass is a mix of equal amounts of SQ-U of Alopecurus pratensis (meadow foxtale), Dactylis glomerata (cocks's foot), Festuca pratensis (meadow fescue),Lolium perenne (English ryegrass), and Phleum pratense(timothy). Each allergen dose was 1.000 SQ-U. A diluent from ALK was used as placebo. Thus the received two injections, one in each groin on three occasions. Patients were randomized in blocks of six, facilitated by Forum Östergötland. An unblinded nurse prepared and marked each syringe with a label providing randomization number, injection number and injection site. ILIT was administered by three clinicians (LA, PR, and UN). Ultrasound-led technique was used whereby a lymph node was punctured with a $27 \mathrm{G}(0.4 \times 40 \mathrm{~mm})$ needle. Histamine- 1 blocker desloratadine tablet $5 \mathrm{mg}$ was given 15 minutes prior to the injections.

\section{Ethics and consent}

The study was approved by the Regional Ethics Committee in Linköping (EPN Dnr 2013/487-31, amendments 2014/55-32 and 2015/296-31). Informed signed consent was obtained from the participants before inclusion.

\section{Primary outcome measures}

Symptoms and drug consumption were primary outcome measures. Symptoms were validated based on the rhinoconjunctivitis total symptom score (RTSS) questionnaire ${ }^{21}$. Drug consumption was measured using an MS questionnaire (see the Methods section in this article's supplementary information). The RTSS and MS were recorded by the patients at the end of the birch pollen season (approximately June $1^{\text {st }}$ ) and at the end of the grass pollen season (approximately September $1^{\text {st }}$ ) before treatment and for the following three and four years altogether. The birch- and grass pollen seasons are quite separate in Sweden.

\section{Safety assessment}

Safety was assessed as the recording of adverse events from the time of the first injection to three years after the last injection. A research nurse called the patients to assess adverse events $2-5$ days after each injection. Safety laboratory parameters were assessed at screening, after the third ILIT injections and after the first pollen season following treatment (Table 2).

\section{Secondary outcome measures}

Effects on quality of life were measured using the rhinoconjunctivitis quality of life questionnaire (RQLQ) 22 recorded as RTSS and MS, see above. Skin prick test reactions (Soluprick SQ Birch and Timothy, ALKAbelló), allergen-specific IgE and allergen-specific IgG4 levels were analyzed (ImmunoCAP ThermoFisher, Uppsala, Sweden) before ILIT and in the fall the following three years. Conjunctival allergen provocation tests (CAPT) ${ }^{23}$ were performed with timothy (Aquagen SQ Timothy, ALK-Abelló) before treatment and after the first pollen season after treatment. Due to lack of extract from the company planned CAPT were not performed after the third pollen season (Table 2).

\section{Immune laboratory methods}

Flow cytometry was used to analyze the CD4+ Th cell population in whole blood from the patients at randomization, and one and three years after completed ILIT. Peripheral blood mononuclear cells obtained from the patients at randomization and one year after ILIT were stimulatedin vitro with birch and timothy allergen. Levels of IL-5, IL-10, IL-13, IFN- $\gamma$, CCL17 and CXCL10 were quantified using Luminex. For 
detailed methods, experimental protocols, and statistical analyses, see the Methods section in this article's supplementary information.

\section{Statistics}

Descriptive statistics for RQLQ, RTSS and MS are presented in medians and percentiles (p25 and p75), and in the graphs with medians and $95 \%$ confidence interval. Paired comparisons over time were calculated with Friedman's test and adjusted with the Bonferroni correction for multiple comparisons. Descriptive statistics for IgE, IgG4, SPT and CAPT are presented in mean values and standard deviation (SD). Paired comparisons over time were calculated with repeated measures ANOVA with Bonferroni confidence interval adjustment. The answers to the 28 questions in RQLQ were explored with an item analysis, rendering a Cronbach's Alpha at 0.933; thus the changes within the different domains of RQLQ were consistent. All analyses above were performed in SPSS version 25 (IBM Corp. Armonk, NY, USA).

All flow cytometry, cytokine and chemokine data were analyzed using GraphPad Prism, version 8.3.1 (GraphPad software, Inc., La Jolla, CA, USA), and non-parametric tests were used. Comparisons at the different time points within the treatment groups were calculated using paired Wilcoxon signed ranks test. Unpaired Mann Whitney $U$ test was used to compare differences between the treatment groups at the different time points. The significance level was set at $\mathrm{p}<0.05$.

\section{Results}

Seventy-four patients were randomized to ILIT with three doses of $0.1 \mathrm{ml}$ of birch- and 5-grass pollen allergen extracts, or either and placebo at monthly intervals. One patient withdrew consent after the first injections and one was lost to follow-up after two injections. Seventy-two patients received all injections (Fig. 1). One patient was lost to follow-up two years after treatment; RQLQ and RTSS had been halved and the patient had not required or used anti-allergic medication. Another patient was lost to follow-up three years after ILIT and two years after treatment showed no improvement in RTSS, MS or RQLQ. Hence, 70 patients remained for analyses three years after treatment.

\section{Symptoms and medication.}

The symptoms measured by the RTSS and MS were significantly reduced three years post ILIT regardless of active allergen during the birch and grass pollen seasons. The reduction was already evident during the first season after ILIT, and the effect was sustained throughout the following three years. When combining all three groups, RTSS was reduced from 12.2 to $7.4(-39 \%)$ and from 11.2 to $6.5(-42 \%)$ three years after treatment during the birch and grass pollen seasons, respectively $(\mathrm{p}<0.05$, Fig. 2c-d, see Table S1 in the Supplementary information). When combining MS data from all three treatment groups together, it was significantly reduced from 9.4 before ILIT to $4.9(-48 \%)$ and from 8.6 to $4.4(-49 \%)$ three years after treatment during the birch and grass pollen seasons, respectively $(\mathrm{p}<0.01$, Fig. 2e-f. For levels of RTSS and MS see Table S1 in the Supplementary information). There were no gender-related differences in improvement (data not shown). The patients receiving ILIT in 2014 and 2015 responded almost in the same way (data not shown).

\section{Adverse events}

A total of 438 injections were given, of which 285 had an active substance containing allergen extract. Mild local pain at the injection site was the most common adverse event (AE). However, on three occasions, patients recorded severe pain from ILIT. One patient had moderate breathing problems without any fall in peak flow 30 minutes after the second ILIT (birch and 5-grass) and received the third treatment without any AEs. One patient experienced breathing problem two hours after physical activity, four days after the first injections, and was relieved with salbutamol inhalations, antihistamine and oral corticosteroids. The remaining injections followed without breathing problems. During the follow-up after ILIT, nine patients reported severe AEs, diverticulitis, miscarriage, burn injury, disc hernia, abdominal pain, hysterectomy, concussion, chest pain, and heart failure but none was judged to be related to ILIT. One patient showed 
hypothyroidism with elevated levels of antithyroid peroxidase, and thyroid-stimulating hormone receptor antibodies diagnosed in 2018, possibly related to the therapy given in 2014 .

\section{Health-related quality of life}

The impact on health-related quality of life as measured by RQLQ was significantly reduced during the birch and grass pollen seasons regardless of active ILIT with birch, 5-grass or both allergens. When combining all three groups, the RQLQ score was significantly reduced from 3.15 to $1.50(-52 \%)$ and from 2.82 to 1.25 $(-56 \%)$ three years after treatment during the birch and grass pollen seasons, respectively ( $<<0.01$, Fig. 2a-b, Table S1 in the Supplementary information).

\section{Conjunctival allergen provocation tests, IgE, IgG4 and skin prick tests}

CAPT with timothy were performed before ILIT and the first year post ILIT, and showed a higher tolerance threshold in patients receiving ILIT with birch and 5 -grass $(\mathrm{p}<0.05)$ and 5 -grass and placebo $(\mathrm{p}<0.05)$, but not significant in patients who received ILIT with birch and placebo $(\mathrm{p}=0.19$, see Table S1 in the Supplementary information). The IgE levels to birch decreased from baseline to three years post ILIT from 25.45 to $17.71 \mathrm{kU} / \mathrm{L}(\mathrm{p}<0.01$ in the group treated with birch and placebo; in the group treated with 5-grass and placebo levels went from 33.18 to $24.42(\mathrm{p}<0.01)$. The levels of IgE to timothy decreased after ILIT with birch and placebo from 16.60 to $11.00 \mathrm{kU} / \mathrm{L}(\mathrm{p}<0.05)$; similar, but non-significant, differences were determined after ILIT with 5-grass and placebo. IgE levels to both birch and timothy decreased slightly after ILIT with both birch and 5-grass (see Table S1 in the Supplementary information). Levels of IgG4 antibodies to birch and timothy remained unchanged in all three treatment groups, except for IgG4 levels to timothy, which increased from in mean 0.36 to $0.44 \mathrm{mg} / \mathrm{L}(\mathrm{p}<0.05)$ after ILIT with birch and 5-grass (see Table S1 in the Supplementary information). Skin prick test for reactivity to birch and timothy allergens remained unchanged during the study period (see Table S1 in the Supplementary information).

\section{Circulating T helper cell subsets}

Flow cytometry data revealed that the proportion of Th1 cells, defined as CD $3{ }^{+} \mathrm{CD} 4{ }^{+} \mathrm{CD} 45 \mathrm{RA}{ }^{-}{ }^{-} \mathrm{Tbet}^{+}$cells, decreased between baseline and three years after treatment in the groups receiving ILIT with birch and placebo and 5 -grass and placebo $(\mathrm{p}<0.05$, Fig. 3a-b). An increase was observed after one year in the group receiving birch and 5 -grass treatment $(\mathrm{p}<0.05$, Fig. 3c). The proportion of Th2 cells, defined as $\mathrm{CD}^{+}{ }^{+} \mathrm{CD} 4{ }^{+} \mathrm{CD} 45 \mathrm{RA}^{-} \mathrm{GATA} 3^{+}$cells, increased three years after treatment in the group receiving birch and placebo ILIT (Fig. 3d, p<0.01) and in the group receiving both treatments (Fig. 3f, p<0.05). No other changes were observed in the group that received 5-grass and placebo ILIT (Fig. 3e). The proportion of Th17 cells, defined as $\mathrm{CD}^{+}{ }^{+} \mathrm{CD} 4{ }^{+} \mathrm{CD} 45 \mathrm{RA}^{-} \mathrm{RORC}^{+}$cells, decreased from baseline to three years post ILIT, independent of treatment (Fig. 3g-i, p <0.05). A significant reduction in Th17 cell frequencies was also observed from one year to three years after ILIT $(\mathrm{p}<0.01)$. The Th17 memory population was significantly higher at baseline in the birch and placebo group than in the other treatment groups $(p<0.001$, Fig. S2a in the Supplementary information). One year after treatment, a higher proportion of Th17 memory cells was observed in patients receiving 5 -grass and placebo ILIT than in the birch and placebo group $(\mathrm{p}<0.05$, Fig. S2b in the Supplementary information). No significant differences between the treatment groups were observed at three years (Fig. S2c in the Supplementary information). The proportion of CD $4{ }^{\mathrm{dim}} \mathrm{CD} 25^{\mathrm{hi}} \mathrm{FoxP} 3{ }^{+}$Tregs (Fig. 4a-c) and activated Tregs, defined as $\mathrm{CD} 3^{+} \mathrm{CD} 4^{+} \mathrm{CD} 45 \mathrm{RA}^{-}$FoxP3 $3^{++}$cells (Fig. 4d-f), significantly increased between baseline and three years, independent of treatment. In contrast, the proportion of resting Tregs, defined as $\mathrm{CD} 3{ }^{+} \mathrm{CD} 4{ }^{+} \mathrm{CD} 45 \mathrm{RA}^{+} \mathrm{FoxP} 3^{+}$cells, was not affected by ILIT (data not shown).

\section{Allergen-induced cytokine and chemokine production}

At baseline and one year post ILIT, peripheral blood mononuclear cells were harvested and stimulated with birch and grass allergen in vitro to measure cytokine and chemokine secretion (see Tables S2 and S3 in the Supplementary information). Both birch and grass allergens induced an increase in IL-5 production after birch and placebo ILIT $(\mathrm{p}<0.05$, Fig. $5 \mathrm{a}$ and $\mathrm{b}$, while IL-5 secretion did not change in the other treatment groups. Increased birch allergen induced IL-10 secretion was also observed after birch and placebo ILIT and 
5-grass and placebo ILIT, whereas no significant change occurred in the birch and 5-grass group (Fig. 5c). ILIT did not affect grass-allergen IL-10 production (Fig. 5d). The spontaneous production of the CCL17 chemokine decreased after 5-grass and placebo ILIT and birch and 5-grass ILIT, but not after birch and placebo ILIT $(\mathrm{p}<0.05$ Fig. 5e).

\section{Discussion}

The current study is the largest double-blind randomized clinical ILIT trial to date. Patients with hay fever due to sensitization to both grass and birch pollen allergens received 5-grass and birch ILIT, 5-grass ILIT (with birch placebo), or birch ILIT (with grass placebo). The study revealed statistically significant clinical efficacy of ILIT in all three treatment groups. Participants reported improvements in RTSS, RQLQ as well as reduced MS in the first pollen season following ILIT. The effects were sustained throughout the following three follow-up seasons. We expected that the clinical efficacy of ILIT would mostly be observed for the targeted allergen, i.e . that patients receiving birch ILIT would not respond well during the grass pollen season and vice versa for patients with 5-grass ILIT. Surprisingly, patients receiving birch ILIT or 5 -grass ILIT reported improved clinical symptoms during pollen seasons for which they had received no treatment. The clinical data were accompanied and supported by immunological changes such as an increase in the proportion of Treg cells and birch allergen included secretion of IL-10 after the treatment, as well as decreased spontaneous production of the Th2 chemokine CCL17. In contrast, the increase in the number of Th2 cells and the increased level of allergen-specific IL-5 production after birch ILIT were unexpected. As clinical improvement was sustained throughout our study, we suggest the results are not only due to a placebo effect, although placebo effects may be very strong in AIT-clinical trials ${ }^{24}$. The clinical effect did not seem to be mediated by allergen-specific IgG4, as these antibody levels were not clearly elevated between pre-ILIT baseline and any of the time points thereafter. AIT efficacy has, however, often been reported to correlate with increased IgG4 ${ }^{6}$. Moreover, in previous grass- or birch-pollen ILIT studies, only moderate or no changes in IgG4 were determined ${ }^{15-19}$, whereas a significant increase in allergen-specific IgG4 was determined after ILIT with cat dander allergen ${ }^{25}$. In contrast, the beneficial clinical responses correlated with an increase in the frequency of Tregs and increased secretion of IL-10. This potentially inhibitory bystander effect of Tregs and IL-10 might explain why the improved clinical outcome was not dependent on the ILIT allergen.

The birch pollen counts in 2014 and 2018 were higher than in 2015, 2016, and 2017. Fifty-five study subjects received ILIT in 2014 and seventeen received ILIT in 2015, so differences in pollen count were leveled out. Patients who received ILIT in 2015 compared well in the following seasons to patients that received ILIT in 2014. Recall bias is limited as the patients recorded their RQLQ, RTSS and MS directly after the birch and grass pollen seasons, which are quite separate in Sweden, after enrollment, before ILIT and three years after, i.e. four years in all. Of seventy-two patients receiving ILIT, only two was lost to follow up after three years.

Most of the AEs were judged as mild or moderate, generally at the allergen injection site, which is also a normal reaction after SCIT ${ }^{26,27}$. However, whereas patients receiving ILIT in the current study reported between none to three AEs, the number of AEs after SCIT can be up to 40-50. In a large study that analyzed AEs in 1,700 patients who had received SCIT, systemic AEs were reported in $3.3 \%$ of the injections 27. Edema and pruritus at the injection site, flush, urticaria, wheezing, dyspnea, eye pruritus, headache, and abdominal pain are common (1-10\%) or very common $(>10 \%)$ with SCIT, whereas oral pruritus, oral edema, rhinitis, headache, ear pruritus, throat irritation, asthma, abdominal pain, urticaria, and fatigue are common or very common with SLIT ${ }^{28}$. ILIT seems to give AEs similar to those with SCIT, but because ILIT only needs three injections, the AEs can be reduced by up to $90 \%$ compared with SCIT.

One limitation of this study is that it had no true placebo group because all the participants received ILIT against, at least, one pollen. This was for ethical reasons as we did not want any patient with rather severe ARC to be without immunotherapy for four years. The approach with "active allergen placebo" was suggested in an ARIA-GA ${ }^{2}$ LEN statement in $2011^{29}$ and supported by a recent SCIT study where dualallergic patients were randomized to receive either grass or birch SCIT. Here, targeted but not untargeted 
allergen rhinoconjunctivitis symptoms were reduced in contrast to our results ${ }^{30}$.

AIT renders significant improvements in rhinoconjunctivitis and conjunctival sensitivity that persist at least seven years after termination of treatment ${ }^{7}$. In addition, AIT can prevent development of asthma ${ }^{31,32}$ and new sensitizations in mono-sensitized patients ${ }^{12,33}$. After an updosing phase of $7-15$ weeks in SCIT, a maintenance phase of three years with injections every six weeks is typically required to render long-term tolerance. This long duration is a major problem with SCIT ${ }^{7}$. For SLIT, $55-82 \%$ of patients were reported to

abandon the treatment before completing the recommended course of therapy ${ }^{34}$. With only three injections over eight weeks, ILIT may overcome the disadvantages of long duration of treatment and poor compliance.

The results of this study add to the hitherto positive studies and suggest that ILIT is effective and safe as treatment for pollen allergy. We will follow the patients in this study for symptoms, health-related quality of life and adverse events in an open study until 2024 (EPN 2017/302-31).

In conclusion, ILIT is an opportunity to make AIT more easily accessible to patients at a lower cost and less risk. There is a need for further studies to establish the optimal dose for efficacy and side effects ${ }^{20}$.

\section{References}

1. Eriksson J, Ekerljung L, Rönmark E, et al. Update of prevalence of self-reported allergic rhinitis and chronic nasal symptoms among adults in Sweden. The clinical respiratory journal. 2012;6(3):159-168.

2. Warm K, Hedman L, Lindberg A, Lötvall J, Lundback B, Rönmark E. Allergic sensitization is agedependently associated with rhinitis, but less so with asthma. The Journal of allergy and clinical immunology. 2015;136(6):1559-1565.e1551-1552.

3. Newson RB, van Ree R, Forsberg B, et al. Geographical variation in the prevalence of sensitization to common aeroallergens in adults: the GA(2) LEN survey. Allergy. 2014;69(5):643-651.

4. Cardell LO, Olsson P, Andersson M, et al. TOTALL: high cost of allergic rhinitis-a national Swedish population-based questionnaire study. NPJ primary care respiratory medicine. 2016;26:15082.

5. Di Bona D, Bilancia M, Albanesi M, Caiaffa MF, Macchia L. Cost-effectiveness of grass pollen allergen immunotherapy in adults.Allergy. 2020.

6. Akdis CA, Akdis M. Mechanisms of allergen-specific immunotherapy and immune tolerance to allergens. The World Allergy Organization journal. 2015;8(1):17.

7. Roberts G, Pfaar O, Akdis CA, et al. EAACI Guidelines on Allergen Immunotherapy: Allergic rhinoconjunctivitis. Allergy.2018;73(4):765-798.

8. Klimek L, Pfaar O, Bousquet J, Senti G, Kundig T. Allergen immunotherapy in allergic rhinitis: current use and future trends. Expert review of clinical immunology. 2017;13(9):897-906.

9. Hoffmann HJ, Valovirta E, Pfaar O, et al. Novel approaches and perspectives in allergen immunotherapy. Allergy.2017;72(7):1022-1034.

10. Dhami S, Nurmatov U, Arasi S, et al. Allergen immunotherapy for allergic rhinoconjunctivitis: A systematic review and meta-analysis.Allergy. 2017;72(11):1597-1631.

11. Arvidsson MB, Löwhagen O, Rak S. Effect of 2-year placebo-controlled immunotherapy on airway symptoms and medication in patients with birch pollen allergy. The Journal of allergy and clinical immunology.2002;109(5):777-783.

12. Durham SR, Penagos M. Sublingual or subcutaneous immunotherapy for allergic rhinitis? Journal of Allergy and Clinical Immunology.2016;137(2):339-349.e310.

13. Senti G, Prinz Vavricka BM, Erdmann I, et al. Intralymphatic allergen administration renders specific immunotherapy faster and safer: a randomized controlled trial. Proc Natl Acad Sci U S A.2008;105(46):1790817912. 
14. Patterson AM, Bonny AE, Shiels WE, 2nd, Erwin EA. Three-injection intralymphatic immunotherapy in adolescents and young adults with grass pollen rhinoconjunctivitis. Annals of allergy, asthma $\mathcal{E}$ immunology : official publication of the American College of Allergy, Asthma, ES Immunology. 2016;116(2):168-170.

15. Schmid JM, Nezam H, Madsen HH, Schmitz A, Hoffmann HJ. Intralymphatic immunotherapy induces allergen specific plasmablasts and increases tolerance to skin prick testing in a pilot study.Clinical and translational allergy. 2016;6:19.

16. Hylander T, Larsson O, Petersson-Westin U, et al. Intralymphatic immunotherapy of pollen-induced rhinoconjunctivitis: a double-blind placebo-controlled trial. Respir Res. 2016;17:10.

17. Hellkvist L, Hjalmarsson E, Kumlien Georen S, et al. Intralymphatic immunotherapy with 2 concomitant allergens, birch and grass: A randomized, double-blind, placebo-controlled trial. The Journal of allergy and clinical immunology. 2018;142(4):1338-1341 e1339.

18. Ahlbeck L, Ahlberg E, Nyström U, Björkander J, Jenmalm MC. Intralymphatic allergen immunotherapy against pollen allergy: A 3-year open follow-up study of 10 patients. Annals of allergy, asthma 83 immunology : official publication of the American College of Allergy, Asthma, ES Immunology. 2018;121(5):626-627.

19. Witten M, Malling HJ, Blom L, Poulsen BC, Poulsen LK. Is intralymphatic immunotherapy ready for clinical use in patients with grass pollen allergy? The Journal of allergy and clinical immunology. 2013;132(5):1248-1252 e1245.

20. Senti G, Freiburghaus AU, Larenas-Linnemann D, et al. Intralymphatic Immunotherapy: Update and Unmet Needs. International archives of allergy and immunology. 2019;178(2):141-149.

21. Devillier P, Chassany O, Vicaut E, et al. The minimally important difference in the Rhinoconjunctivitis Total Symptom Score in grass-pollen-induced allergic rhinoconjunctivitis. Allergy.2014;69(12):1689-1695.

22. Juniper EF, Thompson AK, Ferrie PJ, Roberts JN. Validation of the standardized version of the Rhinoconjunctivitis Quality of Life Questionnaire. The Journal of allergy and clinical immunology.1999;104(2 Pt 1):364-369.

23. Fauquert J-L, Jedrzejczak-Czechowicz M, Rondon C, et al. Conjunctival allergen provocation test : guidelines for daily practice.Allergy. 2017;72(1):43-54.

24. Pfaar O, Agache I, Bergmann K, et al. Placebo effects in allergen immunotherapy - an EAACI Task Force Position Paper.Allergy. n/a(n/a).

25. Freiberger SN, Zehnder M, Gafvelin G, Grönlund H, Kündig TM, Johansen P. IgG4 but no IgG1 antibody production after intralymphatic immunotherapy with recombinant MAT-Feld1 in human. Allergy.2016;71(9):1366-1370.

26. Kowalski ML, Ansotegui I, Aberer W, et al. Risk and safety requirements for diagnostic and therapeutic procedures in allergology: World Allergy Organization Statement. The World Allergy Organization journal. 2016;9(1):33.

27. Schiappoli M, Ridolo E, Senna G, et al. A prospective Italian survey on the safety of subcutaneous immunotherapy for respiratory allergy. Clinical and experimental allergy : journal of the British Society for Allergy and Clinical Immunology. 2009;39(10):1569-1574.

28. Aasbjerg K, Dalhoff KP, Backer V. Adverse Events During Immunotherapy Against Grass Pollen-Induced Allergic Rhinitis - Differences Between Subcutaneous and Sublingual Treatment. Basic Clin Pharmacol Toxicol. 2015;117(2):73-84.

29. Bousquet J, Schünemann HJ, Bousquet PJ, et al. How to design and evaluate randomized controlled trials in immunotherapy for allergic rhinitis: an ARIA-GA(2) LEN statement. Allergy.2011;66(6):765-774. 
30. Wagenmann M, Worm M, Akboga Y, Karjalainen M, Hohlfeld JM. Randomized immunotherapy trial in dual-allergic patients using "active allergen placebo" as control. Allergy. 2019;74(8):1480-1489.

31. Jacobsen L, Niggemann B, Dreborg S, et al. Specific immunotherapy has long-term preventive effect of seasonal and perennial asthma: 10-year follow-up on the PAT study. Allergy. 2007;62(8):943-948.

32. Valovirta E, Petersen TH, Piotrowska T, et al. Results from the 5-year SQ grass sublingual immunotherapy tablet asthma prevention (GAP) trial in children with grass pollen allergy. The Journal of allergy and clinical immunology. 2018;141(2):529-538.e513.

33. Pajno GB, Barberio G, De Luca F, Morabito L, Parmiani S. Prevention of new sensitizations in asthmatic children monosensitized to house dust mite by specific immunotherapy. A six-year follow-up study. Clinical and experimental allergy : journal of the British Society for Allergy and Clinical Immunology. 2001;31(9):1392-1397.

34. Bender BG, Oppenheimer J. The special challenge of nonadherence with sublingual immunotherapy. The journal of allergy and clinical immunology In practice. 2014;2(2):152-155.

35. Hedenström H, Malmberg P, Fridriksson HV. Reference values for lung function tests in men: regression equations with smoking variables. Upsala journal of medical sciences. 1986;91(3):299-310.

36. Hedenström H, Malmberg P, Agarwal K. Reference values for lung function tests in females. Regression equations with smoking variables.Bulletin europeen de physiopathologie respiratoire.1985;21(6):551-557.

37. Miyara M, Yoshioka Y, Kitoh A, et al. Functional delineation and differentiation dynamics of human CD4+ T cells expressing the FoxP3 transcription factor. Immunity. 2009;30(6):899-911.

38. Jenmalm MC, Björksten B, Macaubas C, Holt BJ, Smallacombe TB, Holt PG. Allergen-induced cytokine secretion in relation to atopic symptoms and immunoglobulin $\mathrm{E}$ and immunoglobulin $\mathrm{G}$ subclass antibody responses.Pediatric allergy and immunology : official publication of the European Society of Pediatric Allergy and Immunology.1999;10(3):168-177.

39. Forsberg A, Abrahamsson TR, Björksten B, Jenmalm MC. Pre- and post-natal Lactobacillus reuteri supplementation decreases allergen responsiveness in infancy. Clinical and experimental allergy : journal of the British Society for Allergy and Clinical Immunology.2013;43(4):434-442.

TABLE 1 Baseline characteristics by treatment group, presented as mean and standard deviation

\begin{tabular}{llll}
\hline & Birch + 5-grass & Birch + placebo & 5-grass + placebo \\
\hline $\mathrm{n}$ & 23 & 25 & 26 \\
Female & $11(49 \%)$ & $12(48 \%)$ & $12(46 \%)$ \\
Mean age at study start & $38.1 \pm 10.2$ & $36.8 \pm 10.0$ & $34.8 \pm 9.9$ \\
Min/max age at study start & $19.3 / 53.3$ & $21.0 / 53.4$ & $19.5 / 51.4$ \\
Other sensitizations * & $2.6 \pm 1.1$ & $1.8 \pm 1.3$ & $2.7 \pm 0.9$ \\
FEV1\% & $90.5 \pm 10.0$ & $97.3 \pm 9.7$ & $91.8 \pm 7.6$ \\
FENO ppb & $24.5 \pm 27.0$ & $22.2 \pm 17.8$ & $22.6 \pm 15.7$ \\
\hline
\end{tabular}

* SPT with mugwort, cat, dog, horse, Dermatophagoides pteronyssinus, Dermatophagoides farinae, Cladosporium, Alternaria andAspergillus extracts (Soluprick SQ Birch and Timothy, ALK-Abelló, Hørsholm, Denmark)

FEV1\%: forced expiratory volume at the end of the first second, percent of predicted value ${ }^{35,36}$

FENO-ppb: fraction of exhaled nitric oxide in parts per billion 


\section{TABLE 2 Scheme of schedule procedures}

\begin{tabular}{|c|c|c|c|c|c|c|}
\hline & Screening & Randomi-zation & Intervention & Intervention & Intervention & Follow- \\
\hline & Visit 1 & Visit 2 & Visit 3 & Visit 4 & Visit 5 & Visit 6 \\
\hline Informed consent & $\mathrm{x}$ & & & & & \\
\hline Phys. examination & $\mathrm{x}$ & $\mathrm{x}$ & & & & $\mathrm{x}$ \\
\hline Blood pressure, pulse, PEF & $\mathrm{x}$ & $\mathrm{x}$ & $\mathrm{x}$ & $\mathrm{x}$ & $\mathrm{x}$ & $\mathrm{x}$ \\
\hline Safety tests ${ }^{1}$ & $\mathrm{x}$ & $\mathrm{x}$ & & & & $\mathrm{x}$ \\
\hline Immunol. tests $^{2}$ & $\mathrm{x}$ & $\mathrm{x}$ & & & & $\mathrm{x}$ \\
\hline SPT & $\mathrm{x}$ & $\mathrm{x}$ & & & & $\mathrm{x}$ \\
\hline Lung function & $\mathrm{x}$ & $\mathrm{x}$ & & & & $\mathrm{x}$ \\
\hline U-HCG & & $\mathrm{x}$ & $\mathrm{x}$ & $\mathrm{x}$ & $\mathrm{x}$ & \\
\hline RQLQ, RTSS, MS ${ }^{3}$ & $\mathrm{x}$ & $\mathrm{x}$ & & & & $\mathrm{x}$ \\
\hline $\mathrm{CAPT}^{4}$ & & $\mathrm{x}$ & & & & $\mathrm{x}$ \\
\hline $\mathrm{AE}$ & & $\mathrm{x}$ & $\mathrm{x}$ & $\mathrm{x}$ & $\mathrm{x}$ & $\mathrm{x}$ \\
\hline Concom. med & $\mathrm{x}$ & $\mathrm{x}$ & $\mathrm{x}$ & $\mathrm{x}$ & $\mathrm{x}$ & $\mathrm{x}$ \\
\hline Diary teaching ${ }^{5}$ & $\mathrm{x}$ & & & & $\mathrm{x}$ & $\mathrm{x}$ \\
\hline ILIT & & & $\mathrm{x}$ & $\mathrm{x}$ & $\mathrm{x}$ & \\
\hline Tel contact ${ }^{6}$ & & & $\mathrm{x}$ & $\mathrm{x}$ & $\mathrm{x}$ & \\
\hline
\end{tabular}

Visit 1: Pre-ILIT, pre-season. Visit 2: pre-ILIT, post-season. Visit 3; 7-35d post visit 2. Visit 4: 28-42d post visit 3. Visit 5: 28-42d post visit 4. Visit 6: Fall year 1. Visit 7: Fall year 2. Visit 8: Fall year 3.

1 Haematology: leukocytes, leukocyte differentiation (neutrophil, eosinophils, basophils, lymphocytes) haemoglobin and platelets at visits 1, 2, and 6. Coagulation blood tests at visits 1 and 2 (PK-INR and APTT).

2 Total IgG and subsets (lgG1-G4), total IgE, allergen-specific IgE and IgG4, and other immunological tests.

${ }^{3}$ RTSS, RQLQ and MS were measured after the birch pollen season (approx. Jun 1st) and after the grass pollen season (approx. Aug 1st)

4 The conjunctival challenge tests (CAPT) with timothy were performed according to the EAACI guidelines 23. Due to lack of extract from the company planned CAPT were not performed after the third pollen season

5 The diary had space for description of the adverse events (AEs) since the last visit.

6 Two to five days after visit 3-5, telephone contact was made considering symptoms after the allergen injections.

All inclusion and exclusion criteria were checked at visits 1-5.

PEF: peak expiratory flow, SPT: skin prick test, U-HCG: urine human chorionic gonadotropin (only in females). ILIT: Intralymphatic immunotherapy.

FIGURE 1 Flow chart 


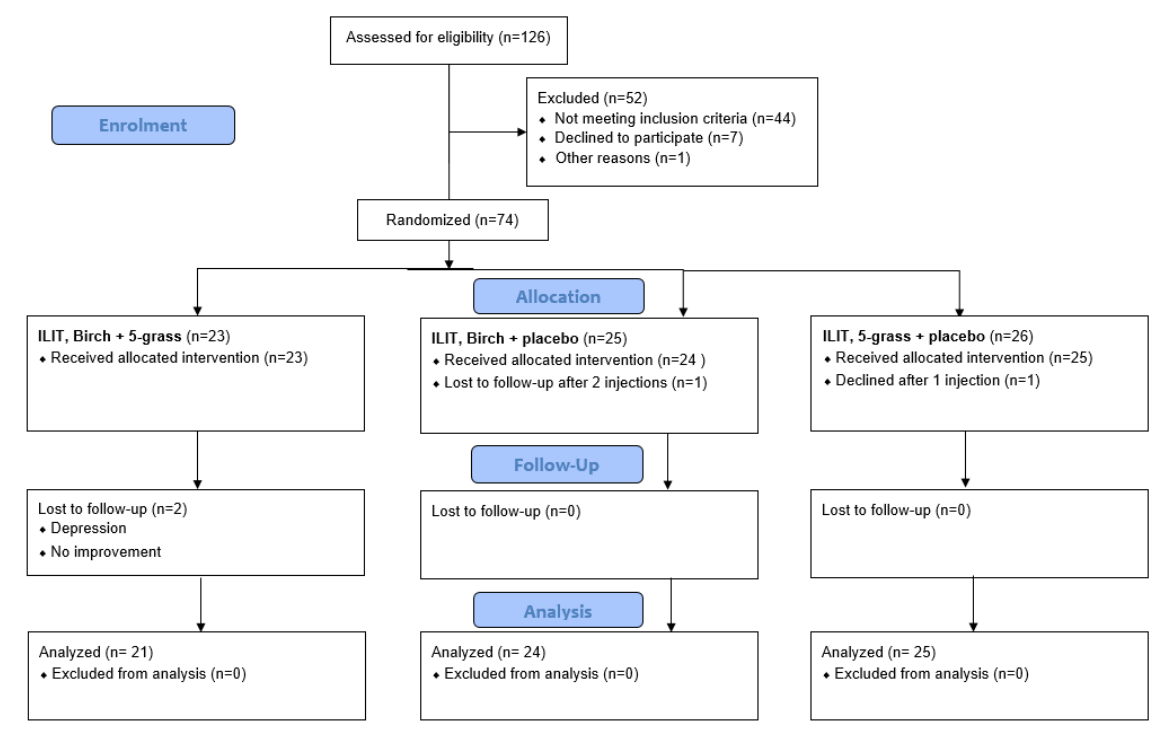

FIGURE 2

a

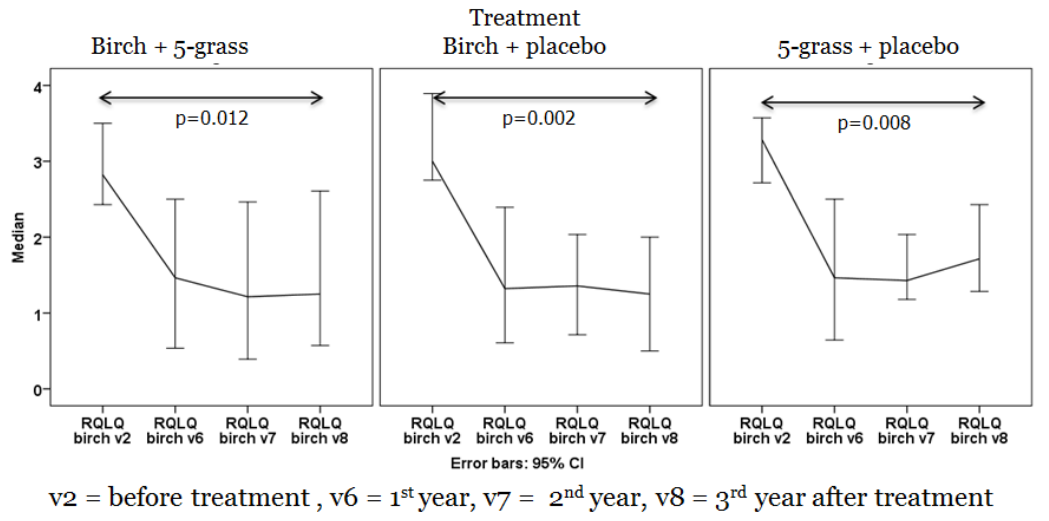

b

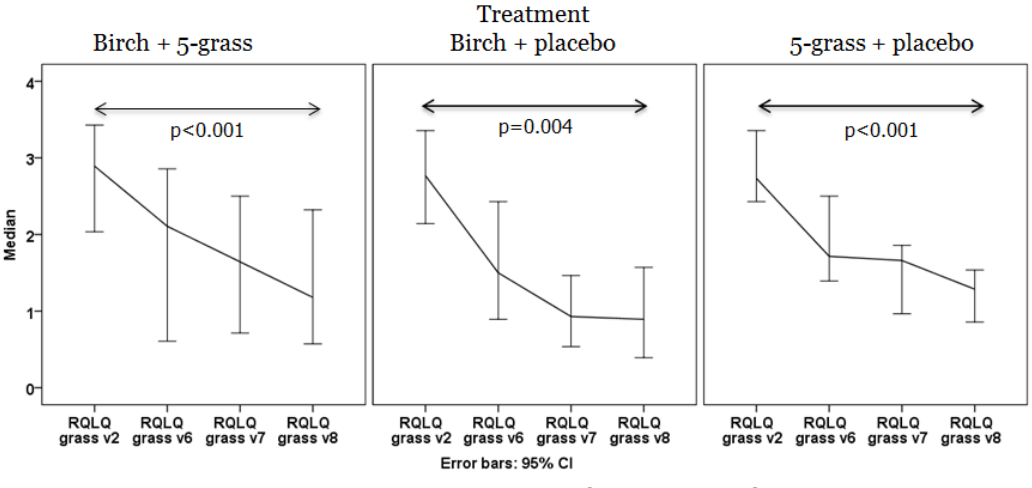

$\mathrm{v} 2=$ before treatment, $\mathrm{v} 6=1^{\text {st }}$ year, $\mathrm{v} 7=2^{\text {nd }}$ year, $\mathrm{v} 8=3^{\text {rd }}$ year after treatment 


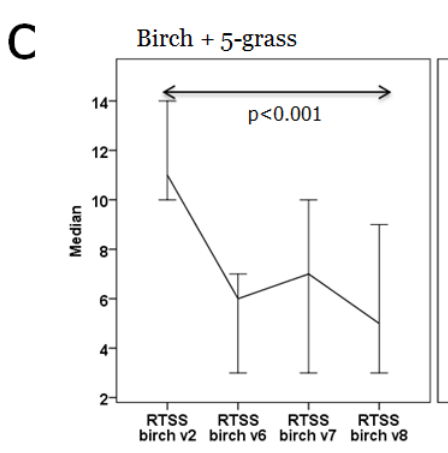

Treatment Birch + placebo

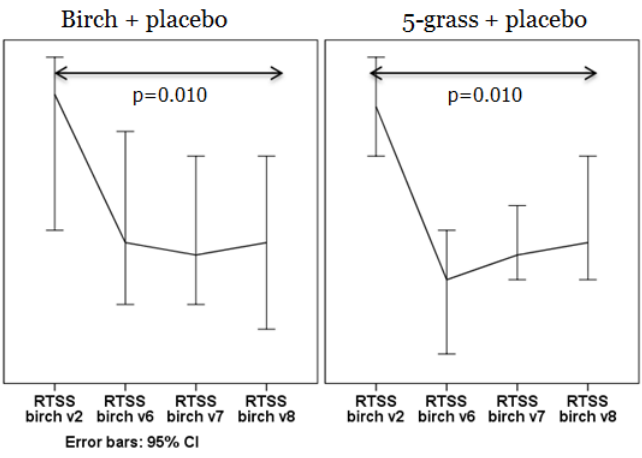

$\mathrm{v} 2=$ before treatment, $\mathrm{v} 6=1^{\text {st }}$ year, $\mathrm{v} 7=2^{\text {nd }}$ year, $\mathrm{v} 8=3^{\text {rd }}$ year after treatment

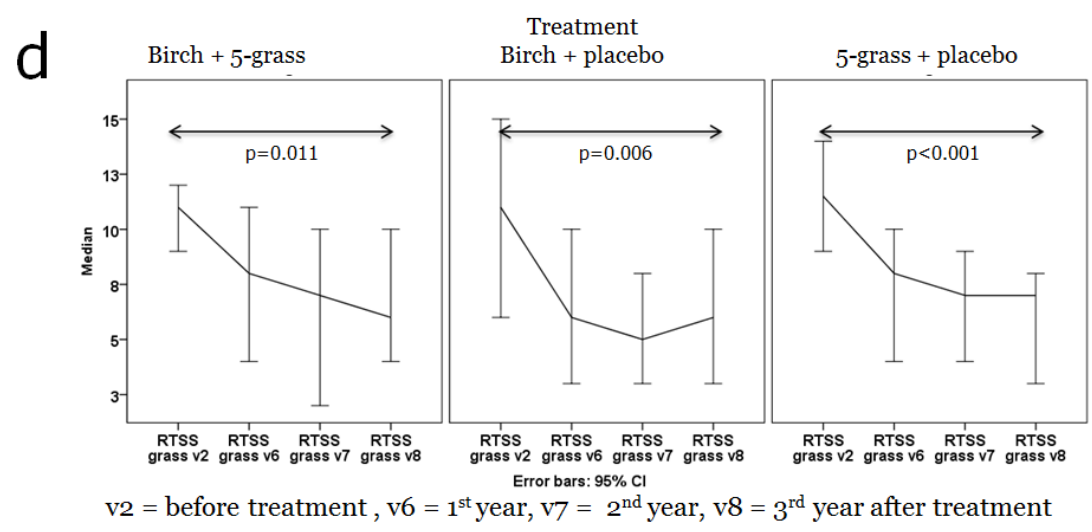

e

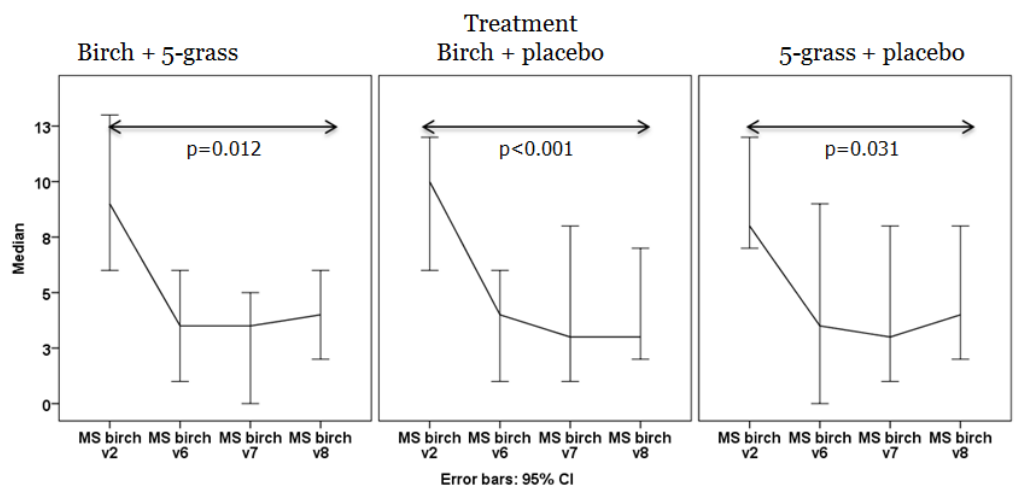

$\mathrm{v} 2=$ before treatment, $\mathrm{v} 6=1^{\text {st }}$ year, $\mathrm{v} 7=2^{\text {nd }}$ year, $\mathrm{v} 8=3^{\text {rd }}$ year after treatment 


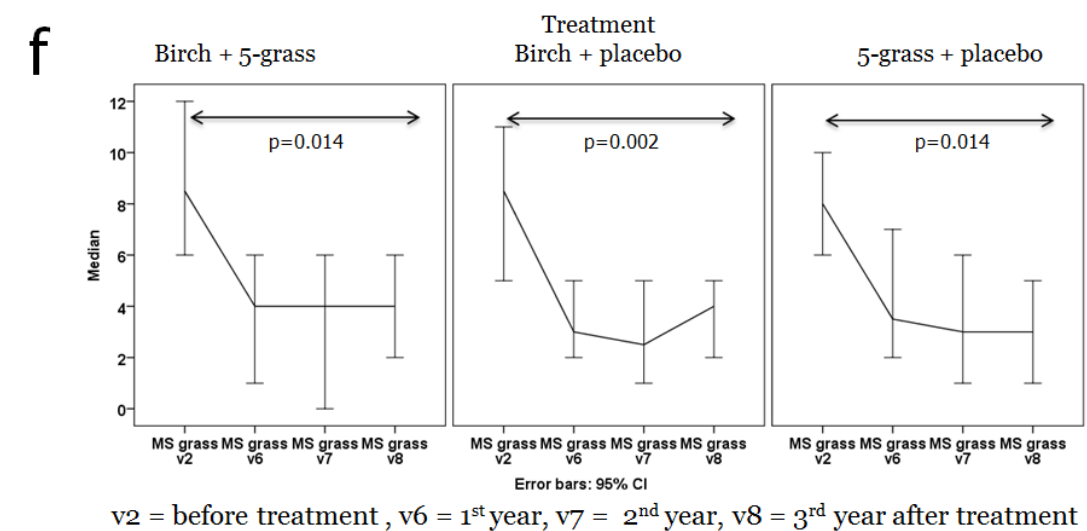

FIGURE 2 Quality of life measured as RQLQ birch pollen seasons (a) and grass pollen seasons (b). Symptoms measured as RTSS birch pollen seasons (c) and grass pollen seasons (d). Medication measured as MS (see under Method section in the Online Repository)) birch pollen seasons (e) and grass pollen seasons (f).

RQLQ: Rhinoconjunctivitis Quality of Life Questionnaire, RTSS: Rhinoconjunctivitis Total Symptom Score, MS: Medication Score

\section{FIGURE 3}


T helper 1 cells
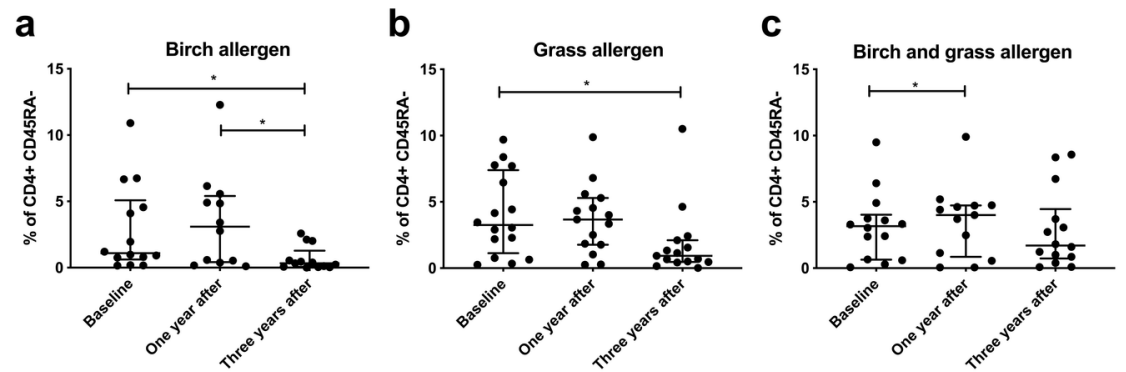

T helper 2 cells
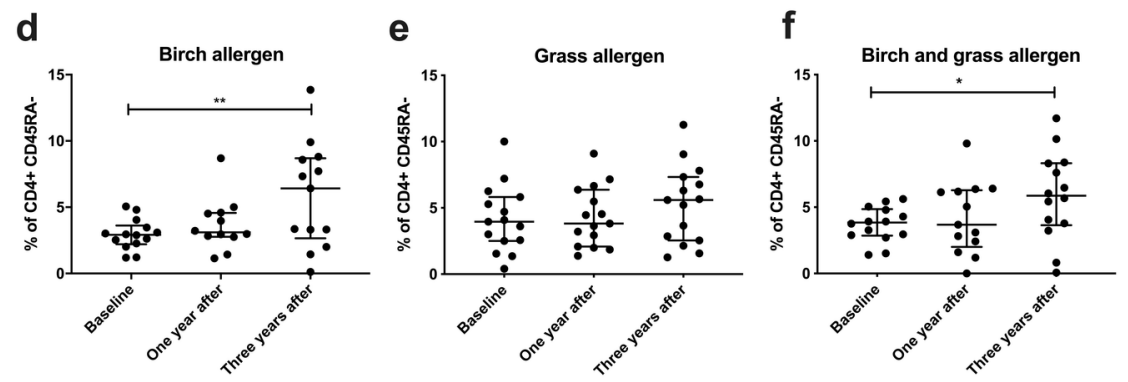

T helper 17 cells
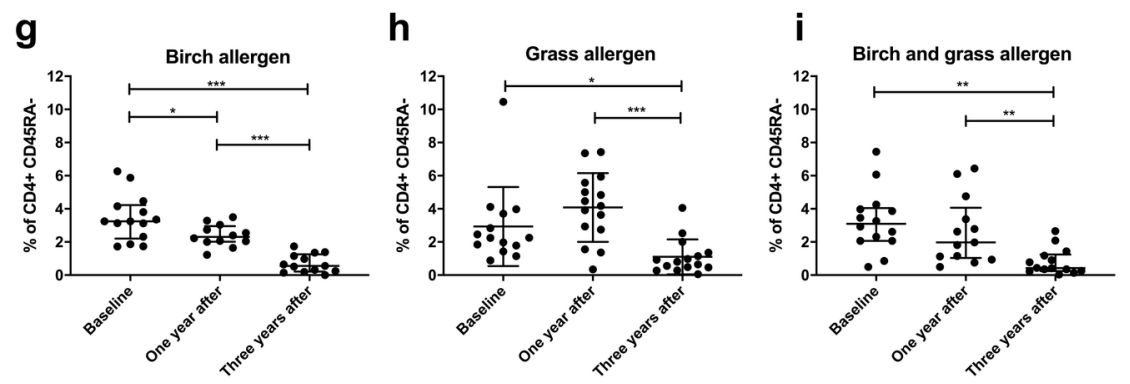

FIGURE 3 Proportion (\%) of the $\mathrm{T}$ helper (Th) cells in the CD4 ${ }^{+} \mathrm{CD} 45 \mathrm{RA} \mathrm{A}^{-}$memory populations after intralymphatic immunotherapy with birch and/or 5-grass allergen. Blood samples were collected at three timepoints: screening, one year after, and three years after treatment had finished. The proportion of Th1 cells, defined as $\mathrm{CD} 3{ }^{+} \mathrm{CD} 4{ }^{+} \mathrm{CD} 45 \mathrm{RA}^{-} \mathrm{Tbet}^{+}$cells, decreased over time after treatment in the groups receiving birch and placebo (a) and in the group receiving grass and placebo (b). A slight increase was observed between baseline and one year after treatment in the group receiving both birch and grass allergen (c). The proportion of Th2 cells, defined as $\mathrm{CD} 3^{+} \mathrm{CD} 4{ }^{+} \mathrm{CD} 45 \mathrm{RA}^{-} \mathrm{GATA} 3^{+}$cells, increased between baseline and three years after treatment in the group receiving birch (d), not in the group receiving grass and placebo. Furthermore, Th2 cells increased three years after treatment in the group receiving both treatments (f). The proportion of Th17 cells, defined as $\mathrm{CD}^{+}{ }^{+} \mathrm{CD} 4{ }^{+} \mathrm{CD} 45 \mathrm{RA}^{-} \mathrm{RORC}^{+}$cells, decreased between all timepoints in the treatment group receiving birch and placebo $(\mathrm{g})$. The treatment groups receiving grass and placebo treatment (h) and birch and grass treatment (i) had similar changes; the proportion decreased between baseline and three years after treatment. A decrease was also observed between one year after and three years after treatment had finished. $\mathrm{p}^{*}<0.05 ; \mathrm{p}^{* *}<0.01 ; \mathrm{p}^{* * *}<0.001$ from Wilcoxon signed rank tests. The lines indicate median and interquartile range (IQR, $25^{\text {th }}$ and $75^{\text {th }}$ percentile values).

FIGURE 4 


\section{T regulatory cells}
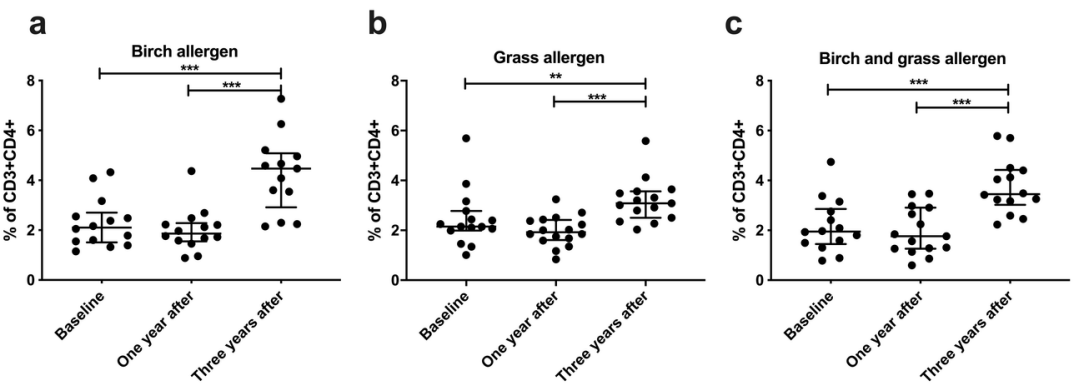

\section{Activated T regulatory cells}
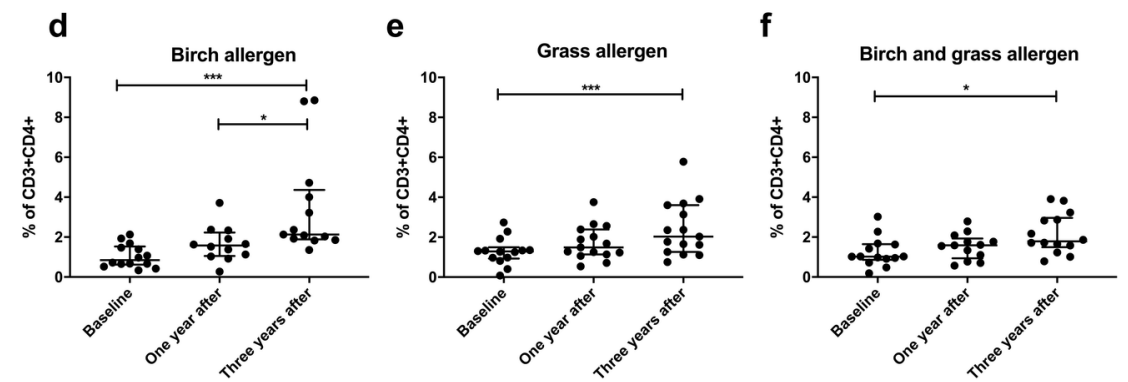

FIGURE 4 Proportion (\%) of the T regulatory (Treg) cells in the $\mathrm{CD} 3{ }^{+} \mathrm{CD} 4^{+}$populations after intralymphatic immunotherapy with birch and/or 5-grass allergen. Blood samples were collected at three timepoints: screening, one year after, and three years after treatment had finished. The proportion of Treg cells, defined as $\mathrm{CD} 4{ }^{\mathrm{dim}} \mathrm{CD} 25^{\mathrm{hi}} \mathrm{FoxP} 3^{+}$cells, increased over time in all treatment groups (a-c). The same trend was observed in the activated Treg cell population, defined as CD3 ${ }^{+} \mathrm{CD} 4^{+} \mathrm{CD} 45 \mathrm{RA}^{-} \mathrm{FoxP} 3^{++}(\mathrm{d}-\mathrm{f}) . \mathrm{p}^{*}<0.05$; $\mathrm{p}^{* *}$ $<0.01 ; \mathrm{p}^{* * *}<0.001$ from Wilcoxon signed rank tests. The lines indicate median and interquartile range (IQR, $25^{\text {th }}$ and $75^{\text {th }}$ percentile values).

\section{FIGURE 5.}


a

Birch induced IL-5 production

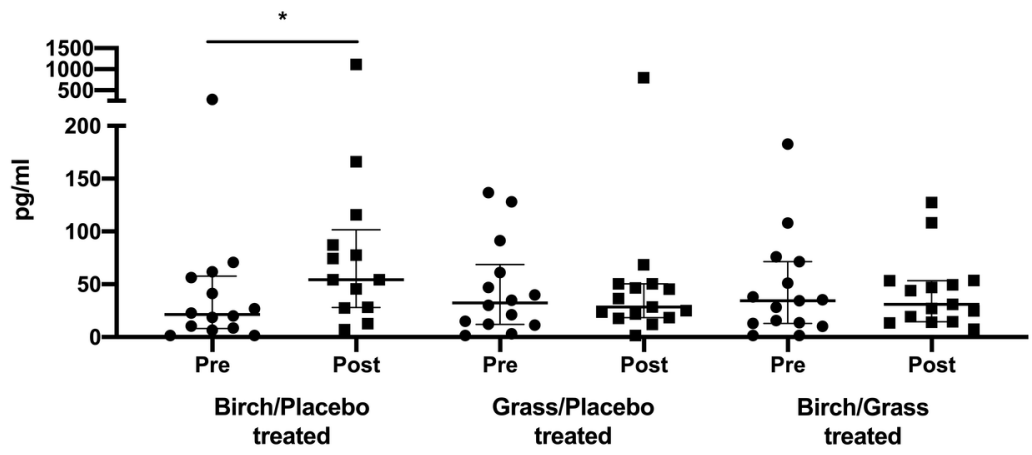

b

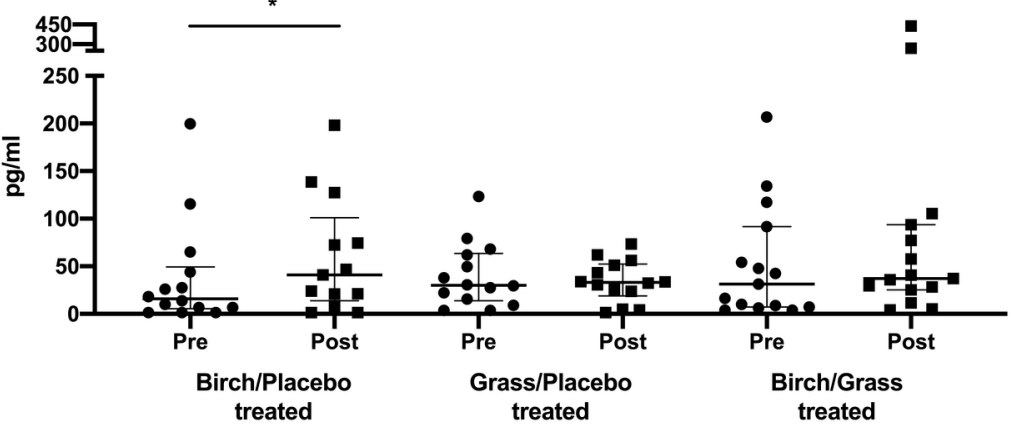

C

Birch induced IL-10 production

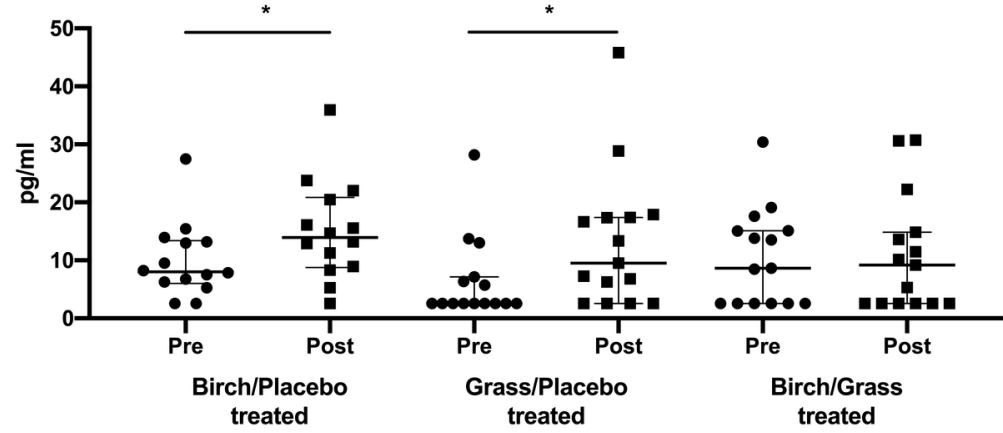

d

Grass induced IL-10 production

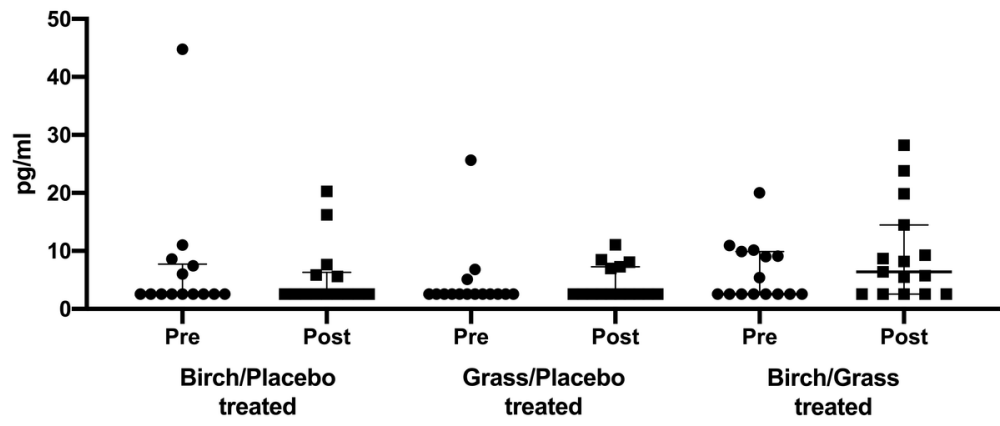

e

Spontaneous CCL17 production

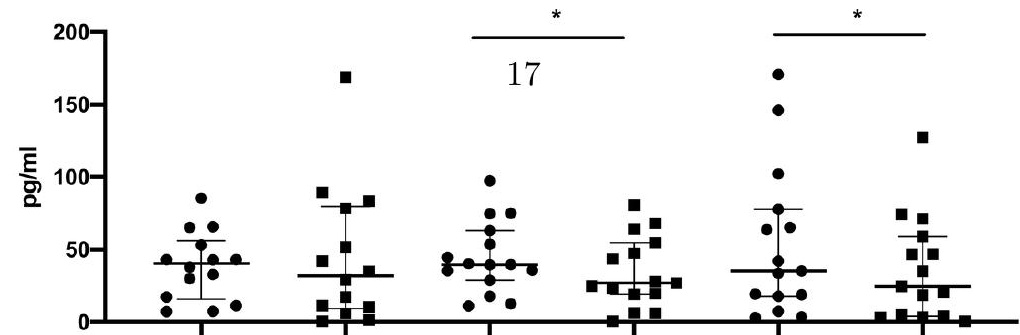


FIGURE 5 Spontaneous and allergen-induced cytokine and chemokine production after intralymphatic immunotherapy with birch and/or 5-grass. a) birch induced IL-5 production b) grass induced IL-5 production, c) birch induced IL-10 production, d) grass induced IL-10 production and e) spontaneous CCL17 production. $\mathrm{p}^{*}<0.05$ from Wilcoxon signed rank tests. The lines indicate median and interquartile range (IQR, $25^{\text {th }}$ and $75^{\text {th }}$ percentile values). 